\title{
IMPACTO DA REEDUCAÇÃO POSTURAL GLOBAL NO TRATAMENTO DA INCONTINÊNCIA URINÁRIA DE ESFORÇO FEMININA
}

\author{
Maria Celina Martins fozzatti*, Paulo Palma, Viviane Herrmann, Miriam Dambros \\ Trabalho realizado na Universidade Estadual de Campinas - UNICAMP, Campinas, SP
}

\section{*Correspondência:}

Av. João Erbolato, 1215

Jd. Chapadão

Campinas - SP

Cep 13070-071

celinafozzatti@terra.com.br

\section{RESUMO}

OBjetivo. Avaliar os efeitos da reeducaçãa postural global (RPG) nos sintomas de incontinência urinária de esforço (IUE) e a qualidade de vida em um grupo de mulheres incontinentes.

Métodos. Ensaio clínico aberto, tipo antes e depois, prospectivo, não randomizado. Foram selecionadas 26 mulheres com queixa clínica e estudo urodinâmico compatível com IUE, do Ambulatório de Uroginecologia do Hospital das Clínicas da UNICAMP. A idade média foi de 50,76 anos (23 a 72 anos). Todas as mulheres foram avaliadas por anamnese, exames clínicos e uroginecológico, avaliação postural. Todas foram submetidas a tratamento com RPG, em sessões individuais de 50 minutos semanais por três meses e quinzenais por mais três meses. As pacientes foram submetidas a posturas de alongamento propostas pela técnica, em que se coloca em tensão as cadeias musculares responsáveis pela postura estática e estrutura do corpo. $\mathrm{O}$ trabalho ativo da paciente nas correções, aliado à atuação do fisioterapeuta, levam ao reequilibrio das tensões musculares e reestruturação do corpo, o que pode favorecer as funções, especificamente as relacionadas ao assoalho pélvico. Ao término do tratamento, e após seis meses, as pacientes foram reavaliadas através do questionário de impressão geral de melhora, dos domínios relacionados à IUE do King's Health Questinnaire: impacto da incontinência, em que se avaliam os prejuízos na qualidade de vida; percepção geral da saúde, qual a classificação que a paciente dá para sua saúde no momento e a presença do sintoma de perda por esforço; avaliação funcional do assoalho pélvico (AFA) e número de trocas de protetores diários.

Resultados. No término do tratamento, quatro pacientes (16\%) estavam curadas, dezoito (72\%) melhoraram significativamente e três (I2\%) falharam. Após seis meses, seis pacientes (24\%) estavam curadas, 16 (64\%) melhoraram e três ( $\mid 2 \%$ ) falharam $(p<0,00 I)$. Ao avaliarmos a qualidade de vida das pacientes, observamos melhora significativa $(p<0,05)$ em todos os domínios questionados, destacando-se percepção geral da saúde, impacto da incontinência e número de episódios de perda. A avaliação do funcional do assoalho pélvico (AFA) e do teste do absorvente (número de troca/dia) também mostrou melhora significativa $(p<0,001)$ nos diferentes tempos de coleta.

ConcLusão. Os resultados mostram que a RPG pode ser uma alternativa para o tratamento da IUE.

UNITERMOS: Incontinência urinária de esforço. Reeducação postural global (RPG).

\section{INTRODUÇÃO}

A incontinência urinária $(I U)$ é definida pela International Continence Society (ICS) como qualquer perda involuntária de urina', sendo que a incontinência urinária de esforço (IUE) é a causa mais comum de IU entre as mulheres. A IUE é definida como a perda involuntária de urina no esforço ou exercício, tosse ou espirro, representando problema social e psicológico 1,2,3. Alguns estudos mostram que nas mulheres adultas da comunidade a prevalência varia de I5\% a $45 \%$ e nas institucionalizadas, acima de 50\% 4,5 . A mulher sofre, no decorrer dos anos, alterações posturais que podem desestruturar sua bacia pélvica, causadas por fatores como gravidez, parto, obesidade, alterações nas curvas fisiológicas da coluna vertebral, atividades esportivas, atividades profissionais, efeito da ação da força da gravidade, dentre outros. O corpo busca, então, novo equilíbrio, muitas vezes com danos às funçõ̃es orgânicas. Existe ligação entre a pelve e a cavidade abdominal, sendo que as variações de pressão na cavidade abdominal são transmitidas para as estruturas pélvicas. Para proteção dessas estruturas, existe um diafragma pélvico, bem como ligações de tecido conjuntivo e ligamentoso, cuja finalidade é manter as vísceras intra-abdominais acima da porção fechada do assoalho pélvico (AP) ${ }^{6}$.

O tratamento da IUE pela RPG busca realinhar os eixos ósseos, eliminar pontos de tensão exagerada e, por outro lado, flacidez dos músculos, reorganizar a tensão nas cadeias musculares e colocar o centro de gravidade do corpo no centro da bacia. Como conseqüência, espera-se desenvolver uma estrutura pélvica presente no esquema corporal, com maior consciência e maior atividade dos músculos do AP e aumentar a capacidade de controle ativo eficaz das funções esfincterianas. Este estudo teve como objetivo avaliar o impacto da RPG sobre os sinais e sintomas de IUE e na qualidade de vida de um grupo de mulheres incontinentes. 


\section{Métodos}

Tratou-se de um ensaio clínico aberto, tipo antes e depois, prospectivo, não randomizado. Foram selecionadas 26 pacientes atendidas no Ambulatório de Uroginecologia do Hospital das Clínicas da Universidade Estadual de Campinas, todas com queixa clínica de IUE. A idade média das pacientes foi de 50,76 anos (23 e 72 anos). A média do tempo de queixas foi de 7,32 anos (I a 23 anos). As pacientes foram incluídas no estudo após assinatura do Termo de Consentimento Livre e Esclarecido. A avaliação incluiu anamnese, exame clínico, avaliação postural, exames de Urina I e urocultura e estudo urodinâmico.

Foram excluídas do estudo as pacientes que apresentaram distopias de grau III e IV; fizessem uso de drogas anticolinérgicas; com queixa clínica de bexiga hiperativa; e, ao estudo urodinâmico, apresentassem contrações não inibidas do detrusor e/ou pressão de perda ao esforço (PPE) abaixo de $60 \mathrm{cmH} 2 \mathrm{O}$.

As ferramentas utilizadas foram o questionário de qualidade de vida King's Health Questionnaire, ${ }^{7}$ traduzido e validado para o português, com dez domínios, em que se investiga o impacto da IUE na qualidade de vida nos aspectos: atividades físicas, psicológicos, emocionais, relações pessoais, atividades sociais, sexuais, qualidade de sono, medidas de prevenção para as situações de perda e escala de sintomas. O questionário foi aplicado na avaliação inicial, no término do tratamento e no seguimento de seis meses, na forma de entrevista, pela presença no grupo de pacientes com baixo grau de instrução, o que dificultou a compreensão. A pontuação dos domínios foi feito pela "Raw Sacale" (0-100); diário miccional de três dias em que se colheu a freqüência miccional, o número e o fator causal dos episódios de perda e o número de troca de protetores devido às perdas, teste do absorvente ${ }^{8} \mathrm{e}$ avaliação funcional do assoalho pélvico (AFA), realizado sempre pelo mesmo fisioterapeuta, em todas as pacientes, através do toque digital intravaginal no assoalho pélvico, na avaliação inicial, no término do tratamento e no seguimento de seis meses, como segue?:

\section{Avaliação funcional do assoalho pélvico (AFA)}

Paciente deitada em decúbito supino, joelhos flexionados. Orientá-la a contrair os músculos do assoalho pélvico, como interrompendo o fluxo urinário. Grau 0: Sem função perineal objetiva; Grau I: Função perineal objetiva ausente, contração reconhecível somente à palpação; Grau 2: Função perineal objetiva débil, contração fraca à palpação; Grau 3: Função perineal objetiva presente e resistência não opositora à palpação; Grau 4: Função perineal objetiva presente e resistência opositora não mantida à palpação; Grau 5: Função perineal objetiva presente e resistência opositora mantida mais do que cinco segundos à palpação.

Foram realizadas sessões de RPG individuais, semanais, de 50 minutos, durante três meses ${ }^{10}$. Posteriormente, as sessões passaram a quinzenais, por mais três meses, sendo que as pacientes tiveram acompanhamento por um período de seis meses após término do tratamento.

O método da RPG trabalha com o alongamento das cadeias musculares propostas por PE Souchard, através de posturas de alongamento. $O$ método tem como princípio o trabalho em globalidade, em que o objetivo é reequilibrar os grupos musculares entre si, especialmente os músculos estáticos, começando pelo alongamento dos mais encurtados, que são responsáveis pela deformaçãa ${ }^{10}$. São oito famílias de posturas, em decúbito supino com ângulo aberto ou fechado das articulações coxo-femurais, com os braços abertos ou fechados, e posturas em carga, sentada e em pé. As posturas em decúbito permitem melhor abordagem manual do fisioterapeuta e as posturas em carga são mais ativas e proprioceptivas. Sempre se inicia o trabalho em posições de menor tensão da cadeia em questão, evoluindo no grau de alongamento, respeitando a condição individual de cada paciente, até chegar ao máximo de evolução. No término da sessão, a paciente é colocada em pé, onde os desequilíbrios são corrigidos manualmente pelo fisioterapeuta e ativamente pelo paciente para integração das correções no que se refere ao esquema corporal. A percepção dos músculos do assoalho pélvico (MAP) e sua participação nos movimentos do tronco são incentivados neste momento ${ }^{\prime !}$. Deve-se escolher a combinação de duas posturas numa consulta de RPG, preferencialmente uma em decúbito e uma em carga, sempre após avaliação das retrações, buscando eliminar os desequilíbrios.

\section{Resultados}

Das 26 mulheres selecionadas para o estudo, 25 concluíram o tratamento. No término do tratamento quatro mulheres (16\%) estavam curadas, 18 mulheres (72\%) apresentaram melhora significativa e três ( $12 \%)$ não apresentaram melhora. No seguimento de seis meses, seis mulheres (24\%) apresentaram-se curadas, 16 mulheres (64\%) mantiveram seu grau de melhora e três mulheres ( I 2\%) não apresentaram melhora. Os dados estão distribuídos na Tabela I e ilustrados no Gráfico I.

O Gráfico 2 mostra que no início do tratamento (TO), todas as pacientes apresentavam episódios de perda urinária por esforço e em TI e T2 observa-se 32\% das pacientes sem apresentar perdas e o número de perdas por dia, das que mantinham, diminuiu significativamente.

A evolução do AFA nos três tempos de coleta mostrou que em T0 temos $3,85 \%$ das pacientes com grau zero e grau I; $34,63 \%$ com grau 2; $26,92 \%$ com grau 3; 19,23\% com grau 4 e II,54\% com grau 5. Em TI, observamos mudança significativa na perfil do gráfico, no qual

Tabela I - Variáveis de avaliação subjetiva, intervalos de confiança (IC95\%) e testes de hipóteses, para cada tempo de coleta $(n=25)$

\section{Avaliação subjetiva (melhora ou cura)}

Proporção em TI: 88.0\%;

IC95\%: (75.3; 100.0);

$Z=43,72 ; \quad p<0,001$

Proporção em T2: 88.0\%;

IC95\%: (75.3; 100.0);

$Z=43.72$

$p<0,001$

Ausência de sintomas*

Proporção em TI:32.0\%;

Proporção em T2: 24.0\%;

IC95\%: (13.7; 50.3);

$Z=15.58 ; \quad p<0,001$

IC95\%: (7.3; 40.7);

$Z=11.56$

$p<0,001$

\section{Percentual de melhora**}

Média em TI: 76.9\%; $\quad$ IC95\%: $(64.1 ; 89.7) ; \quad t=|2.4| ; \quad p<0,00 \mid$

Média em T2: 60.8\%; $\quad$ IC95\%: (60.8; 86.8); $\quad t=|1.72 ; \quad p<0,00|$

* teste de hipótese para proporção diferente de zero; ** teste de hipótese para média diferente de zero 


\section{Gráfico I - Avaliação subjetiva: grau de satisfação apresentado pelas pacientes submetidas ao tratamento de} IUE pela técnica da RPG, em dois tempos - final de tratamento e seis meses após $(p<0,00 \mathrm{I})$
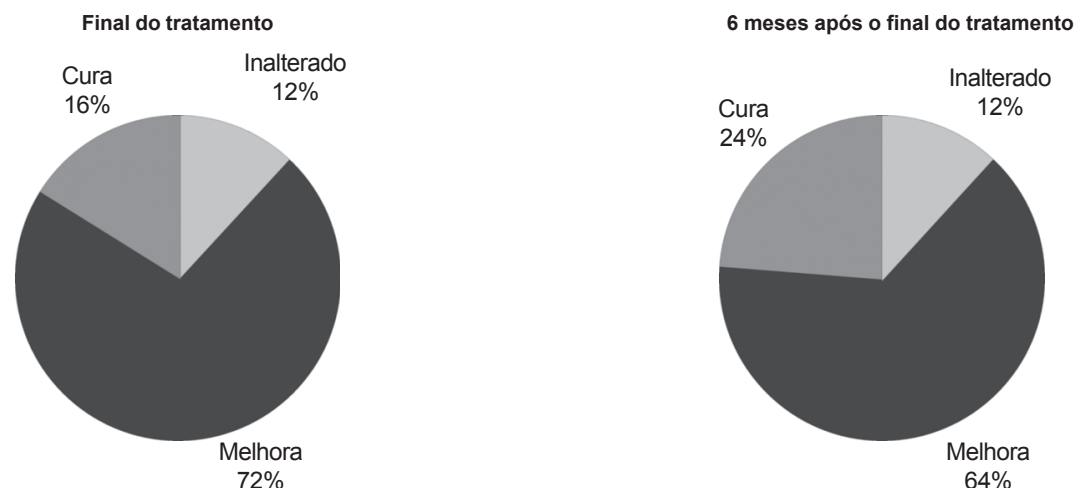

\section{Gráfico 2 - Número de perdas diárias nos diferentes tempos de tratamento $(\mathrm{p}<0,00 \mathrm{I})$}

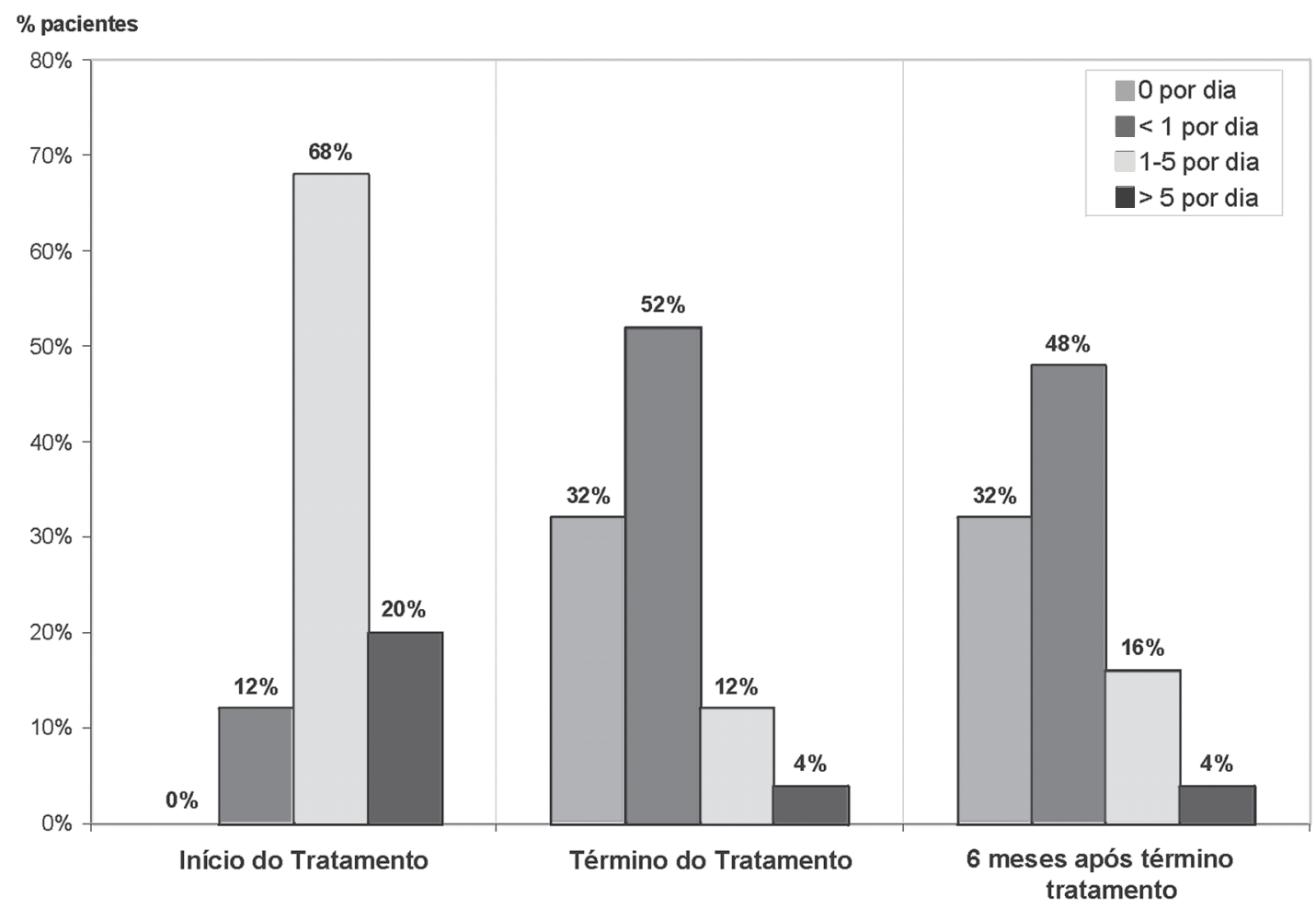

não temos mais pacientes com graus zero, I e 2; 24\% passaram para grau 3; $40 \%$ para grau 4 e $36 \%$ para grau 5 . Em T2, vemos $16 \%$ das pacientes com grau 3; $48 \%$ com grau 4 e $36 \%$ com grau 5 . Ao traçarmos a média de evolução no grau da AFA, temos em T0 a média de 2,88; em TI passou para 4, 12 e em T2 a média foi para 4,20. 0 Gráfico 3 ilustra que houve uma significativa diminuição na troca de absorventes por dia, a partir do final do tratamento.
O Gráfico 4 mostra a variação dos domínios avaliados do King's Health Questionnaire, a percepção geral da saúde, impacto da incontinência e sintoma de perda aos esforços, mostrando melhora significativa em todos eles nos três tempos avaliados $(p<0,05)$.

A análise descritiva e comparativa das variáveis principais entre os tempos de coleta estão distribuídos na Tabela 2. Como a seleção da amostra foi limitada por fatores condicionados à população estudada, 


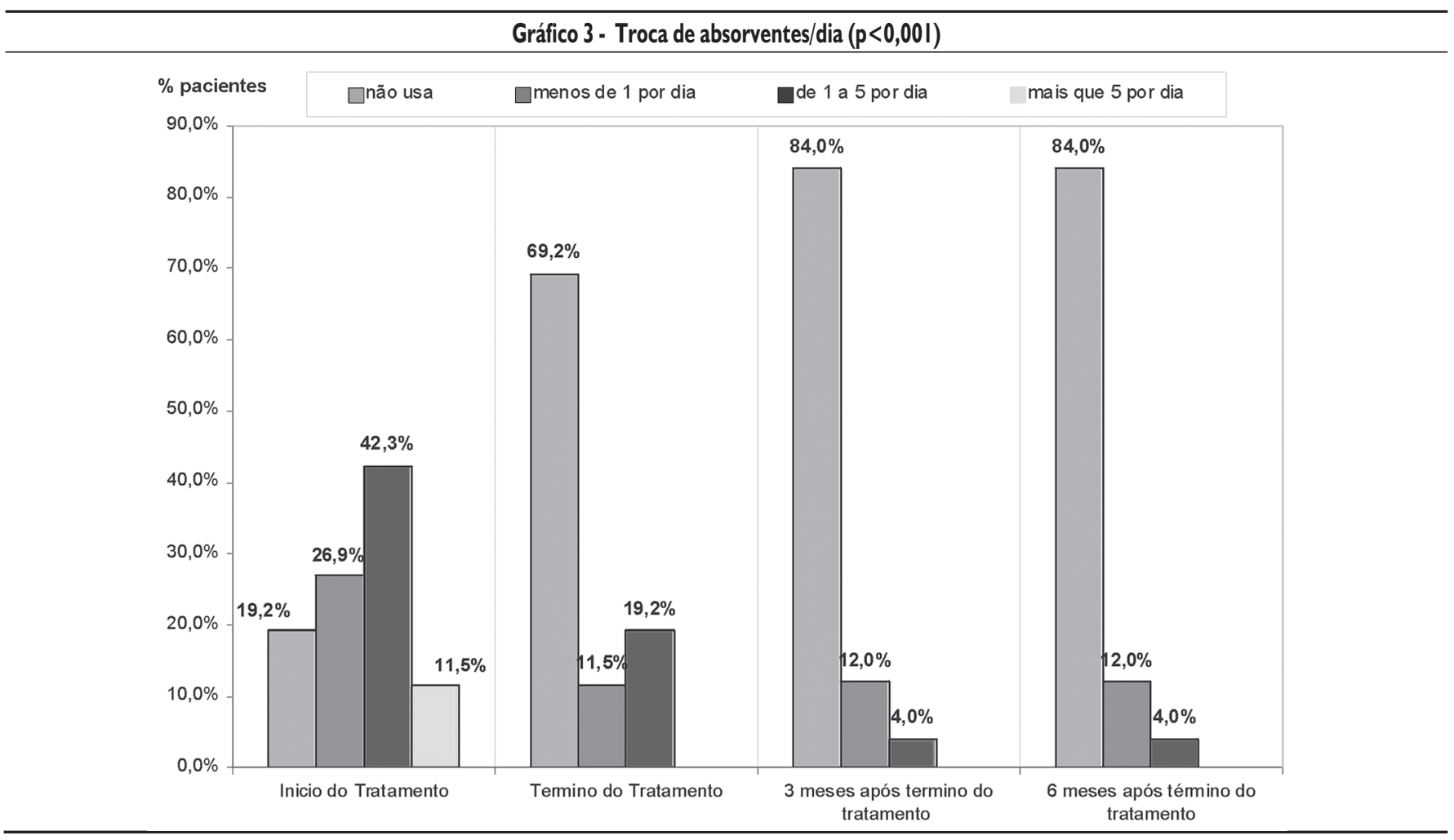

Gráfico 4 - King's Health Questionnaire - Impacto dos sintomas de IUE na qualidade de vida das pacientes antes e após o tratamento co $m$ a RPG

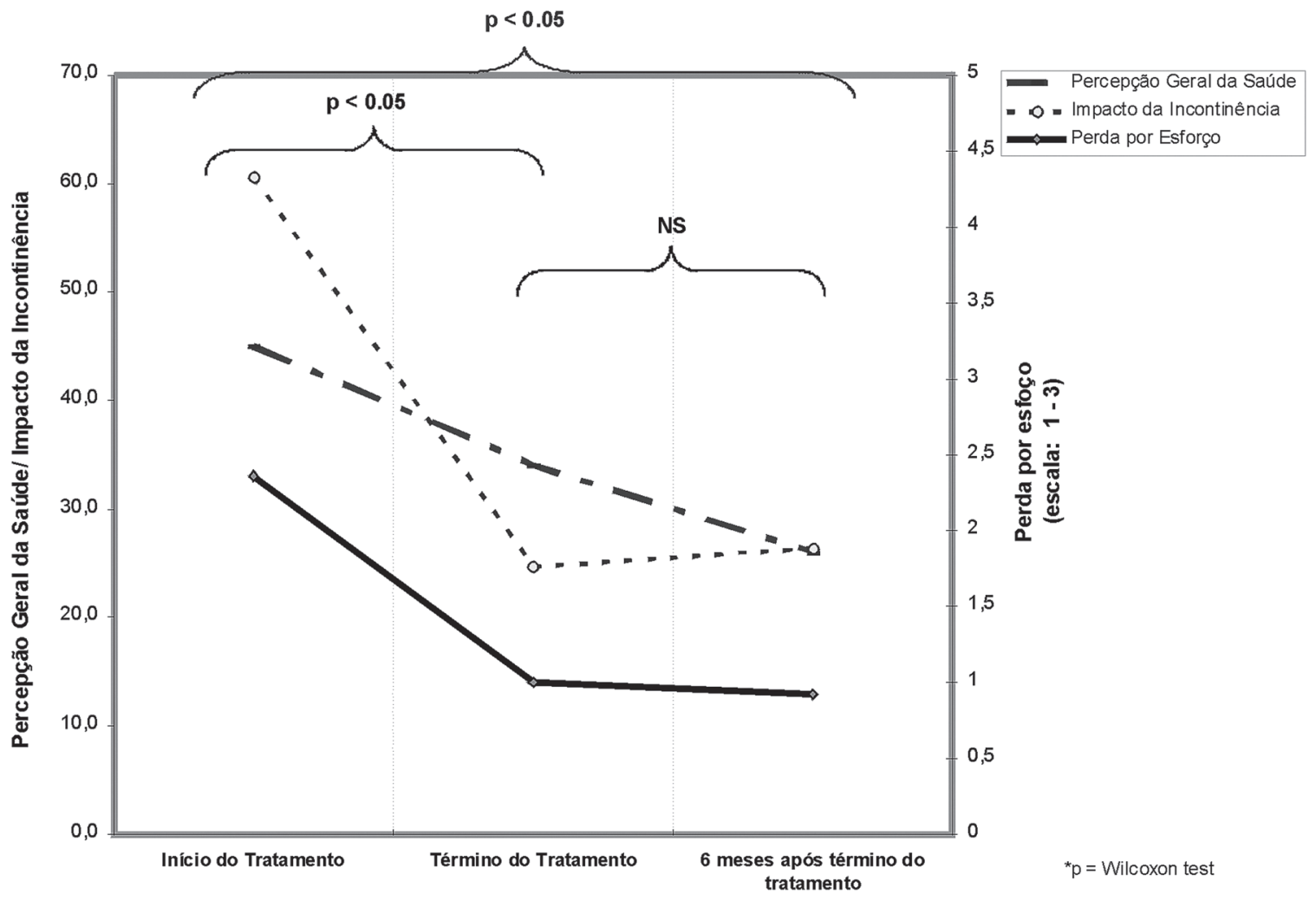




\begin{tabular}{|c|c|c|c|}
\hline Varável & $\begin{array}{l}\text { Teste de } \\
\text { Friedman }\end{array}$ & p-valor & $\begin{array}{c}\text { diferenças } \\
\text { significativas } \\
\text { entre os tempos }\end{array}$ \\
\hline $\operatorname{Perdas}(T 0, T I, T 2)$, & $x^{2}=45.72$ & $p<0,001$ & $\mathrm{~T} 0 \neq T 1 ; \mathrm{T} \mid \neq \top 2$ \\
\hline Pad Use $(T 0, T I, T 2)$ & $x^{2}=34.34$ & $p<0,00 \mid$ & $\mathrm{TO} \neq \top 1 ; \mathrm{T} \mid \neq \top 2$ \\
\hline $\operatorname{AFA}(\mathrm{TO}, \mathrm{TI}, \mathrm{T} 2)$ & $x^{2}=54.73$ & $p<0,001$ & $\mathrm{~T} 0 \neq \mathrm{T} 1 ; \mathrm{T} \mid \neq \top 2$ \\
\hline Percepção geral de melhora & $x^{2}=|0.4|$ & $p<0,005$ & $T 0 \neq T 1 ; T 1 \neq T 2$ \\
\hline Impacto da incontinência & $x^{2}=25.18$ & $p<0,001$ & $T 0 \neq T 1 ; T 1 \neq T 2$ \\
\hline Sintomas-perda por esforço(KHQ) & $x^{2}=28.34$ & $p<0,001$ & $\mathrm{TO} \neq T|; \mathrm{T}| \neq T 2$ \\
\hline
\end{tabular}

como distância, fator socioeconômico e transporte, optamos por calcular o poder da amostra estudada nas principais variáveis do estudo: percepção geral da saúde, impacto da incontinência e incontinência urinaria por esforço, do KHQ, considerando os valores obtidos na primeira e na última avaliação, para comparação de médias num estudo longitudinal, para dados pareados ou relacionados. Os cálculos foram feitos fixando o erro do tipo I em $5 \%$ (ou $a=0,05$ ) e o tamanho da amostra em $n=25 .^{14,15}$ Nas três variáveis utilizadas foram obtidos valores de poder altos: percepção geral da saúde 88\% (beta=0.117); impacto da incontinência 99\% (beta $=0,00 \mathrm{I}$ ) e escore de incontinência de esforço 99\% (beta $=0.00 \mathrm{I}$ ), indicando que o tamanho da amostra coletada é suficiente para as conclusões.

\section{Discussão}

A IUE é uma condição comum que leva a sério comprometimento na qualidade de vida da mulher, com danos sociais, psicológicos e higiênicos. Nygaard et al ${ }^{16}$, mostram que 30\% das mulheres queixamse de perdas urinárias durante exercícios físicos. Isso se deve à constante pressão que esta musculatura sofre durante atividades físicas de alto impacto. O bom equilíbrio da bacia depende da condição postural. A bacia bem posicionada propicia o equilíbrio dos órgãos pélvicos dentro da cavidade abdominal, o que favorece suas funções ${ }^{17}$. O equilíbrio da bacia mostra os ilíacos paralelos entre si, com as cristas ilíacas ânterosuperiores orientadas para frente, os ísquios para baixo, sacro com ângulo de aproximadamente 30\%, medido a partir do platô sacral e a linha horizontal ${ }^{18}$.

Nesta situação, a tensão dos músculos estruturais da bacia pélvica, os pelvitrocanterianos, dos músculos que compõem a massa comum, na região lombar, dos músculos transversos do abdome e dos MAP estão normalizadas. Isto se reflete na adequada transmissão de pressão dentro da cavidade abdominal e atividade de resposta dos músculos do $\mathrm{AP}$ às variações da pressão, favorecendo o processo de continência. Quando as pacientes deste estudo foram avaliadas e fotografadas, mostraram desequilíbrios posturais sugestivos de modificação no ângulo sacral, que na maioria, partiu para posição mais horizontal, o que pode provocar estiramento dos músculos do AP, comprometendo sua função. Bo et al ${ }^{19}$, mostraram que durante a contração desses músculos há um movimento do coccix para frente, em direção ao púbis. Isso pode sugerir haver relaçãa entre a posição do sacro e a atividade desses músculos. Após analisadas segundo o padrão postural, as pacientes foram submetidas as posturas que favoreceram o reequilíbrio das cadeias musculares, eliminando as tensões ou flacidez do(s) músculo(s) causador(es) do desvio do osso, buscando a normalização global e favorecendo as funções orgânicas.

Portanto, o equilíbrio entre lordose lombar, músculos transverso do abdome, responsáveis pela manutenção da pressão intra-abdominal e a forma cilíndrica do tronco, a mobilidade do diafragma torácico, mais importante músculo da função respiratória e responsável pelas variações da pressão intra-abdominal e o eixo de rotação das articulações coxo-femurais, são os princípios básicos do tratamento de IUE pela RPG. A aceitação ao tratamento foi excelente, visto que as pacientes ganharam auto-conhecimento do seu corpo e passaram a ter mais domínio sobre suas estruturas. Isso se reflete no gesto do dia-a-dia, fazendo com que os MAP participem dos movimentos do tronco e membros inferiores e mantenham atividade permanente de ajuste de tensão, mantendo sua forma. Isso se confirma quando, nos resultados obtidos, observamos que o índice de cura aumentou no seguimento de seis meses. Também podemos notar a evolução do AFA, que mostrou melhora a partir do término do tratamento. Como resultado, o impacto desta condição na qualidade de vida foi diminuído, mostrando que as pacientes retomaram atividades que foram comprometidas pela situação de perda, como podemos verificar na análise dos domínios do King's Health Questionnaire, nos diferente tempos.

A RPG tem como vantagem ser um tratamento não-invasivo, não apresentar riscos à paciente e com a possibilidade de outras intervenções, caso necessário. Além disso, melhora a qualidade de vida das pacientes pela modificação do esquema corporal, melhora do auto-conhecimento do corpo, melhora do gesto, o que submete à menor sobrecarga das estruturas e, portanto, diminui a predisposição a lesões e aumentos desnecessários da pressão intraabdominal, protegendo os MAP. A melhora da consciência e domínio de suas estruturas corporais favorece o uso dos músculos do AP no gesto do dia- a-dia, isto é, o uso funcional automático desses músculos, o que os mantêm saudáveis. O tratamento com o treinamento dos músculos do AP mostra melhora nos sintomas de IUE, como têm descrito a literatura, porém condiciona a mulher à prática permanente dos exercícios para manutenção dos resultados, o que é uma desvantagem, pois, a curto prazo, esta prática poderá ser abandonada. Porém, temos ainda poucos estudos comparando estas duas técnicas. Não se encontra tampouco na literatura trabalhos que mostrem resultados no tratamento da IUE com a correção dos desequilíbrios posturais. A literatura menciona freqüentemente o fator postural nas IUE ${ }^{20}$, mas não são encontrados estudos com propostas de métodos de tratamento específico na abordagem desta condição. Este é o primeiro estudo que aborda a IUE através da RPG. O seguimento deste grupo de pacientes permitirá comprovar os resultados a longo prazo. 


\section{Conclusão}

Considerando os resultados obtidos, conclui-se que a RPG induziu à melhora significativa dos sintomas de IUE em relação à satisfação da paciente, AFA, diário miccional e qualidade de vida neste grupo de mulheres incontinentes.

\section{Conflito de interesse: não há}

\section{SUMMARY}

IMPACT OF GLOBAL POSTURAL REEDUCATION FOR TREATMENT OF FEMALE STRESS URINARY INCONTINENCE

OBBECTINE: To evaluate the effect of global postural reeducation(GPR) on stress urinary incontinence (SUI) and quality of life in SUI female patients

METHODS: The study design was a prospective non-randomized clinical trial. Twenty-six patients with symptoms of SUI were selected from the Urogynecology Outpatient Clinics of the State University of Campinas (Unicamp), state of São Paulo, Brazil. Age ranged from 23 to 72 years old (mean 50.8). All women were submitted to anamnesis, physical exam, postural evaluation and urodynamic testing. Patients were treated by the GPR in individual 50 minute sessions weekly for three months and twice a month for the next three months. All patients were re-evaluated at the end of treatment and six months later by means of General Impression of Improvement, Incontinence Impact, General Perception of Health, Functional Evaluation of the Pelvic Floor, Number of Leaking Episodes and Pad Use.

RESULTS: At the end of treatment 4(16\%) of the patients were cured, $18(72 \%)$ had improved significantly and 3 (12\%) failed. At 6 months, 6 (24\%) were cured, $16(64 \%)$ improved and $3(12 \%)$ failed $(p<0.001)$. Quality of Life questionnaires presented significant improvement $(p<0.05)$ in all domains, with emphasis on General Perception of Health, Incontinence Impact and number of leaking episodes. The Functional Evaluation of the Pelvic Floor and Pad Use also presented significant $(p<0.001)$ improvement.

CONCLUSION: These results may demonstrate that GPR is an efficient alternative for treatment of stress urinary incontinence. [Rev Assoc Med Bras 2008; 54(I): 17-22]

KEY wORDS: Stress urinary incontinence. Global postural reeducation (GPR).
2. Guri R, Hannestad YS, Sandvik H, Hunskaar S. A community based epidemiological survey of female urinary incontinence: the Norweigian EPICONT Study. J Clin Epidemiol. 2000;53: I I 50-7.

3. Bo KS. Evaluation of female pelvic-floor muscle function and strength. Phys Ther. 2005;85:269-82.

4. Berghmans LCM, Hendriks HSM, Bö K, Hay-Smith EJ, De Bie RA, Van Waalmijk J, Van Doorn ESC. Conservative treatment of stress urinary incontinence in women: a systematic review of randomized clinical trials. Br J Urol. 1998;82: |8|-9|.

5. Guarisi T, Pinto Neto AM, Osis MJ, Pedro AO, Paiva LH, Faundes A. Urinary Incontinence among climateric Brazilian Women: Household Sourvey. Rev Saúde Pública 200 I;35:428-35.

6. Wallach S, Ostergard D. Anatomia pélvica femina. In: D'Ancona CAL, Rodrigues Netto Jr N. Aplicaçoões clínicas da urodinâmica; 3aed. São Paulo: Atheneu; 200 I. p. I26-38.

7. Tamanini JTN, D'Ancona CAL, Botega NJ, Rodrigues Netto Jr N. Validação do "King's Health Quetionnaire" para o português em mulheres com Incontinência urinária. Rev Saúde Pública 2003;37:203-I I .

8. Ghoniem GM, Van Leeuwen JS, Elser D, Freedmen RM, Zhao YD, Yalcin I, et al. For Duloxetine /Pelvic Floor Muscle Training Clinical Trial Group. J Urol. 2005; 173: 1647-53.

9. Ortiz OC, Gutnisky R, Nunez FC, Cortese G. Valoracion dinamica de la disfuncion perineal de clasification. Bol Soc Latinoam Uroginecol Cir Vaginal. 1994, 1:7-9.

10. Souchard PE. Reeducação postural global: o método do campo fechado. São Paulo: Ícone Editora; 1986. Cap. 4, p.7I-4.

I I. Souchard PE. RPG: fundamentos da reeducação postural global princípios e originalidade São Paulo: É Realizações; 2003. p.33-40.

12. Siegel S. Estatísitca não-paramétrica para as ciências do comportamento. São Paulo: MacGraw-Hill; 1975.

13. Conover WJ. Practical nonparametric statistics. New York: Jonh Wiley \& Sons; 1971.

14. Pearson ES, Hartley HO. Biometria tables for statisticians. $3^{\text {rd }}$ ed. Cambridge: Cambridge University Press, 2003.

15. Hulley SB, Cummings SR Designing clinical research. Baltimore: Williams \& Wilkings; 1988.

16. Nygaard I, Delancey JOL, Arnsdrf L. Exercise and incontinence. Obstet Gynecol. 1990;75:848-5I.

17. Etienne MA, Watman MC. Disfunções sexuais femininas São Paulo: LMP Editora; 2006. p.67

18. Bienfait M. Fisiologia da terapia manual: São Paulo: Summus Editorial; 1989. p. 141 , p. $171-2$

19. Bö K. Physiotherapy to treat genuine stress incontinence. Int Cont Surv. 1996:6:2-8

20. Wells TJ, Brink CA, Diokno AC, Wolfe R, Gillis GL. Pelvic muscle exercise for stress urinary incontinence in erderly women. J Am Geriatr Soc. 1991;39:785-91.

\section{REFERÊNCIAS}

I. Abrams P, Cardoso L, Fall M, Griffiths D, Rosier P, Ulmsten U, et al. The standardization of terminology of lower urinary tract function: report fron the stardardization of termonology sub-committee of the International Continence Society. Urology 2003;61:3-49.

Artigo recebido: 07/02/07

Aceito para publicação: 10/08/07 